\title{
Advances in anesthesia technology are improving patient care, but many challenges remain
}

\author{
D. John Doyle ${ }^{1,2^{*}}$, Ashraf A. Dahaba ${ }^{3}$ and Yannick LeManach ${ }^{4,5}$
}

\begin{abstract}
Although significant advances in clinical monitoring technology and clinical practice development have taken place in the last several decades, in this editorial we argue that much more still needs to be done. We begin by identifying many of the improvements in perioperative technology that have become available in recent years; these include electroencephalographic depth of anesthesia monitoring, bedside ultrasonography, advanced neuromuscular transmission monitoring systems, and other developments. We then discuss some of the perioperative technical challenges that remain to be satisfactorily addressed, such as products that incorporate poor software design or offer a confusing user interface. Finally we suggest that the journal support initiatives to help remedy this problem by publishing reports on the evaluation of medical equipment as a means to restore the link between clinical research and clinical end-users.
\end{abstract}

Advances during the last several decades have led to important improvements in clinical monitoring technology and clinical practice development, not only in patients undergoing surgery [1-6] or in patients being cared for in Intensive Care Units (ICUs) [7-9] but also in ambulatory patients $[10,11]$. These developments have contributed to great improvements in patient safety $[3,5-7$, 12-14]. In addition, anesthesiologists world-wide have developed standards for continuous real-time monitoring of hemodynamics, oxygenation, ventilation, neurological status, urine output, core temperature, degree of neuromuscular blockade, as well as other items, all of which have also contributed significantly to patient safety [15-17].

Several other innovative developments have also contributed to improving the quality of perioperative care. Checklists, proven to be particularly valuable in the aerospace industry, are now in common use in the operating room and elsewhere [18-22]. For example, in a landmark study by Haynes et al. [23], a surgical death

\footnotetext{
* Correspondence: djdoyle@hotmail.com

${ }^{1}$ Cleveland Clinic Lerner College of Medicine of Case Western Reserve University, Cleveland, Ohio, USA

2Department of General Anesthesiology, Cleveland Clinic Abu Dhabi, Abu

Dhabi, UAE, PO Box 112412, Abu Dhabi, UAE

Full list of author information is available at the end of the article
}

rate of $1.5 \%$ before the introduction of a surgical checklist fell to $0.8 \%$ after, with an inpatient complication rate dropping from a baseline of $11 \%$ down to $7 \%$ after introduction of the checklist. New approaches to clinical airway management such as airway algorithms [24, 25], video laryngoscopy [26-31], extubation catheters [3234] and advanced supraglottic airway devices [35-38] are also protecting patients from injury.

In the realm of perioperative cardiac monitoring, the use of conventional and 3D- echocardiography $[39,40]$ now allows for real-time monitoring of valvular function, ventricular filling, cardiac contractility and other hemodynamic parameters. Additionally, hand-held ultrasound machines are changing how bedside examinations are conducted [41-44] (Fig. 1). Clinical early warning algorithms, especially valuable in the perioperative setting to detect the early onset of clinical deterioration, have also proven to be effective in improving patient care [45-49].

Another important development concerns the numerous so called "Depth of anesthesia monitors" such as the bispectral index (BIS) monitor. Historically, bispectral analysis is a standard high-order statistical analysis first used by oceanographers as a measure of time series to study nonlinearity in ocean waves [50]. This was further developed in the 1960s by geophysicist seismologists to 


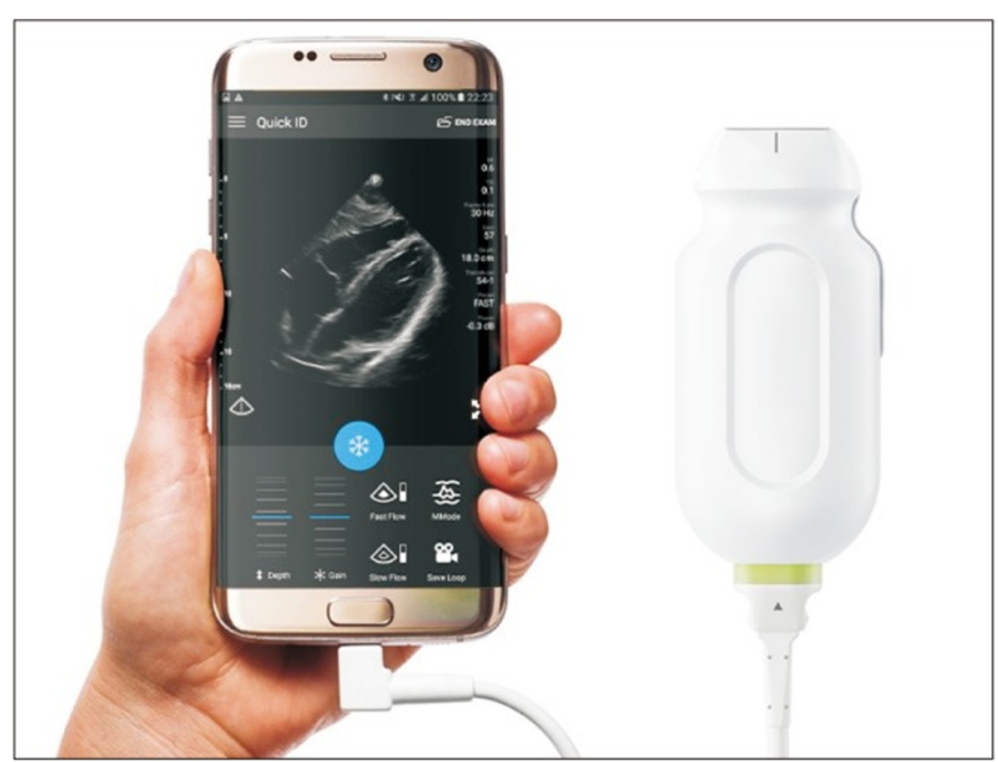

Fig. 1 Ultrasound machines for applications such as echocardiography, regional anesthesia or central line placement have now evolved to the point that they can connected to a smartphone or tablet.Image from Michard F. Smartphones and e-tablets in perioperative medicine. Korean J Anesthesiol. 2017 Oct;70(5):493-499. doi: https://doi.org/10.4097/kjae.2017.70.5.493. PubMed PMID: 29046768; PubMed Central PMCID: PMC5645581. Image used under the terms of the Creative Commons Attribution Non-Commercial License, which permits unrestricted non-commercial use, distribution, and reproduction in any medium, provided the original work is properly cited

study complex seismic waveforms [51]. The first EEGderived monitor, the BIS (Medtronic, Dublin, Ireland) was introduced in 1994 as a monitor of the effects of certain anesthetic/hypnotic agents [52]. To date the complete details of the BIS algorithm have not been published. Scientifically speaking, all that we really know is that the BIS monitor is merely a "black box" headset and the BIS value reflects a "head-related" biosignal that correlates with changes in the biophase effect-site concentrations of certain hypnotic /sedative drugs and thus cannot be considered a "true" reflection of the depth of anesthesia. In other words, the BIS index is a measure of certain drugs' effect and is not a true reflection of the EEG signal nor an independent measure of brain function [53]. In the early years all attempts of the manufacturer for the Food and Drug Administration (FDA) to license the BIS as an "independent uniform depth of anesthesia" monitor failed. For instance, a BIS value of 57 for 1 MAC halothane is significantly higher than the BIS value of 32 for an equipotent 1 MAC sevoflurane [54], and significantly higher than a BIS value of 33 for an equipotent $1 \mathrm{MAC}$ isoflurane [55]. While ketamine provides adequate anesthesia, ketamine administration was reported to paradoxically increase the BIS from 44 to 59 [56].

What is the bispectral index then? The BIS algorithms were derived from EEG changes with incremental doses of certain hypnotic drug(s); isoflurane or propofol while measuring 3 descriptors in volunteers. The BIS index is the weighted sum of 3 sub-parameters; Relative
BetaRatio most influential during light anesthesia, a frequency-domain feature is the EEG spectral power log $\left(\mathrm{P}_{30-47 \mathrm{~Hz}} / \mathrm{P}_{11-20 \mathrm{~Hz}}\right)$, SynchFastSlow predominates during surgical levels of hypnosis, a bispectral-domain feature, is the bispectral power wave band $\log \left(\mathrm{B}_{0.5-47} \mathrm{~Hz}\right.$ $\left./ \mathrm{B}_{40-47 \mathrm{~Hz}}\right)$ and Burst Suppression that detects very deep anesthesia, a time-domain feature combining two separate algorithms: Burst Suppression Ratio that quantifies the extent of isoelectrical silence, and QUAZI suppression index that detects Burst Suppression superimposed on wandering low baseline voltage [57]. None of these disparate descriptors is particular per se; as each has a specific range of influence where they perform best. The BIS analysis uses a proprietary algorithm that allows the 3 different descriptors to sequentially dominate as the EEG changes its character with increasing anesthetics' concentrations. It thus transforms the nonlinear stages of the anesthetic drug effect relative contributions on the EEG into an easy-to-use dimensionless number ranging from 100 (fully awake) to zero (isoelectric EEG) [57].

Obviously, in many instances BIS changes do not truly reflect changing anesthetics' concentrations, as BIS indices would reflect other unrelated EEG events of certain conditions exerting their own EEG effect. Because the BIS is an EEG derived parameter hence anything that would change the EEG would subsequently change the BIS. There is a body of literature of EEG changes of conditions like hypothermia, hypoglycemia, hypovolemia, hypotension, hepatic encephalopathy or physiological 
sleep with the same conditions consequently changing the BIS to the same extent [58].

In the realm of the ever-changing landscape of neuromuscular blockade monitors, older designs are frequently replaced with new devices that are often promoted as technically superior by the manufacturers. Conventional mechanomyography (MMG) is regarded by the Stockholm revision consensus conference [59] as the gold standard for precise quantification of neuromuscular block, as it quantifies the exact force displacement isometric muscle contraction of a preload-restrained thumb in response to electric stimulation at the ulnar nerve [33]. The main obstacle facing its wide clinical use is that the equipment takes time to set up and requires rigid support of the arm.

Over the years, we have seen numerous stand-alone or modular-integrated neuromuscular monitoring devices that quantify the neuromuscular function based on physiological phenomena other than force measurement. The kinemyographic (KMG) device known as ParaGraph [60] (Vital Signs, Totowa, NJ) is no longer available for routine clinical use as the manufacturer has been acquired by CareFusion in 2014 although the neuromuscular transmission module (E-NMT) in the AS/5TM anaesthesia monitor (GE, Helsinki, Finland) [61] is still available. Both quantify the signal generated from thumb adduction via deformation of a piezoelectric film sensor in response to electric stimulation of the ulnar nerve. E-NMT has an additional electromyographic (EMG) transducer that quantifies the evoked compound action potential generated at the thenar eminence.

Another attractive class of devices are acceleromyographic (AMG) monitors. The first commercially available product, TOF-GUARDTM (Organon Teknika, Oss, Netherlands) [62]. now discontinued, has been replaced by a simpler device known as TOF-WatchTM (MIPM, Mammendorf, Germany) [63]. Both measure the acceleration using a piezoelectric sensor attached to a freely moving thumb ("piezo" from the Greek word meaning pressure). Note that according to Newton's second law: force $=$ mass $\mathrm{x}$ acceleration, acceleration is directly proportional to force when mass is constant, so that instead of measuring the evoked force, thumb acceleration can be measured instead. A major impediment of this type of monitoring is the fact that the piezoelectric sensor may not always be "properly aligned" to the optimal plane of the thumb movement. A comprehensive systematic review of acceleromyography by Claudius and Viby-Mogensen described many of the methodological problems facing the technology based on evidence based data of 43 publications [63].

With the manufacturer official announcement of the discontinuance of all the TOF Watch monitors series effective June 2016, this development gave way to a new wave of neuromuscular monitoring devices, namely a novel generation of so-called Tri-axial acceleromyographs. Nowadays the only commercially available acceleromyographs all belong to the new Tri-axial based generation; namely the Stimpod NMS 450 (Xavant, Silverton, Pretoria, South Africa), the TOFscan (Dräger, Lübeck, Germany) and the new modular neuromuscular transducer NMT (Mindray, Shenzhen, China).

The above successes notwithstanding, many vital challenges remain to be addressed by the anesthesia technology community. One of these challenges includes reducing the time interval needed to troubleshoot a malfunctioning electrocardiogram, capnograph, pulse oximeter or some other patient monitor prior to starting an anesthesia case. Another challenge is in the realm of alarms [2, 4, 64]; who has not been irritated when the source of a monitor alarm is completely unapparent or when an asystole alarm occurs despite both a good arterial blood pressure waveform and a high-quality pulse oximeter tracing being present. Such difficulties divert attention from direct patient monitoring as mental effort is expended to address some technical problem. Problems related to poor software design or careless user interface designs have also led to patient harm [65-68].

In view of these concerns, we would like to propose that the journal support initiatives by publishing reports on the evaluation of anesthesia and perioperative equipment [69]. These reports might be made in a manner not dissimilar to information provided by web sites like eopinions.com as well as in specialized magazines like Consumer Reports (which provides evaluations of products such as household appliances) or in reports provided by a number of Personal Computer magazines (focusing on software and hardware products).

We envision two general forms of report. The first kind of report would be an informal "first impressions" description of newly available equipment. These reports - or user's opinions -would frequently make observations concerning ergonomics and equipment usability. Extensive and definite evaluation would not be the primary objective. While necessarily subjective, this information would be valuable to individuals seeking to acquire new equipment. Further, individual feedbacks about a newly available equipment will be of major interest to coordinate clinical evaluations based on structured evaluation protocols in a collaborative effort regrouping clinicians familiar with the equipment.

A second, more formal, kind of report would be supported based on the usual scientific publication presentation. This kind of report would be based on rigorous, reproducible testing methods like those methods used by ECRI (ecri.org) and other testing agencies to produce detailed, formal, laboratory-based assessments. Additionally, these reports would include clinical evaluations based on broadly discussed evaluation protocols 
including modern statistical methods. Because of the nature of the devices under evaluation, research protocols tend to vary in objectives, in design and in quality. Collaborative efforts based on a single well-structured design is sometimes the key to obtain timely clinical evaluations of a new device.

The combination of rapid feedback and qualitative structured evaluations of new equipment will allow anesthesia community to focus on the device of interest in a timely matter. Immediate feedback has the potential to improve the design of new devices, and collaborative evaluation effort is usually the fastest way to obtain sufficient data to reliably draw conclusions about the clinical and economical value of a new device.

We believe the journal can play an important role in this initiative. With a more interactive, a more collaborative, and a more international approach, such an initiative would help restore the link between clinical research and clinical end-users. Further, by quickly producing highquality, clinically relevant evaluations, we believe this initiative could have a long-lasting impact on medical device design as well as ultimately on patient safety.

\section{Abbreviations}

AMG: Acceleromyogram acceleromyographic; BIS: Bispectral index; ECRI: Emergency care research institute; EEG: Electroencephalogram; ICU: Intensive care unit; KMG: Kinemyogram / kinemyographic; NMT: Neuromuscular transmission; TOF: Train of four

\section{Authors' contributions}

All authors contributed substantially to the preparation of this manuscript. All authors read and approved the final manuscript.

\section{Competing interests}

All authors are associate editors of BMC Anesthesiology. The authors declare that they have no other academic or financial conflicts of interest with respect to this manuscript.

\section{Publisher's Note}

Springer Nature remains neutral with regard to jurisdictional claims in published maps and institutional affiliations.

\begin{abstract}
Author details
${ }^{1}$ Cleveland Clinic Lerner College of Medicine of Case Western Reserve University, Cleveland, Ohio, USA. ${ }^{2}$ Department of General Anesthesiology, Cleveland Clinic Abu Dhabi, Abu Dhabi, UAE, PO Box 112412, Abu Dhabi, UAE. ${ }^{3}$ Priv.-Doz. Dr.med.university, Division of General Anaesthesiology, Emergency- and Intensive Care Medicine, Medical University of Graz, Graz, Austria. ${ }^{4}$ Departments of Anesthesia \& Health Research Methods, Evidence, and Impact, Michael DeGroote School of Medicine, Faculty of Health Sciences, McMaster University, 1280 Main Street West Hamilton, Hamilton, ON L8S 4L8, Canada. ${ }^{5}$ Population Health Research Institute, David Braley Cardiac, Vascular and Stroke Research Institute, Perioperative Medicine and Surgical Research Unit, 237 Barton Street East, Hamilton, ON L8L 2X2, Canada.
\end{abstract}

Received: 2 April 2018 Accepted: 3 April 2018

Published online: 13 April 2018

\section{References}

1. Pedersen T, Nicholson A, Hovhannisyan K, Møller AM, Smith AF, Lewis SR. Pulse oximetry for perioperative monitoring. Cochrane Database Syst Rev. 2014 Mar 17;3:CD002013.
2. Imhoff M, Kuhls S, Gather U, Fried R. Smart alarms from medical devices in the OR and ICU. Best Pract Res Clin Anaesthesiol. 2009 Mar;23(1):39-50.

3. Subrahmanyam M, Mohan S. Safety features in anaesthesia machine. Indian J Anaesth. 2013 Sep;57(5):472-80.

4. Johnson KR, Hagadorn Jl, Sink DW. Alarm safety and alarm fatigue. Clin Perinatol. 2017 Sep;44(3):713-28.

5. Tang R, Ranmuthugala G, Cunningham F. Surgical safety checklists: a review. ANZ J Surg. 2014 Mar;84(3):148-54.

6. Staender S, Smith A. Enhancing the quality and safety of the perioperative patient. Curr Opin Anaesthesiol. 2017 Dec;30(6):730-5.

7. Hassan E. Tele-ICU and patient safety considerations. Crit Care Nurs Q. 2018; 41(1):47-59.

8. Bose E, Hoffman L, Hravnak M. Monitoring cardiorespiratory instability: current approaches and implications for nursing practice. Intensive Crit Care Nurs. 2016 Jun;34:73-80.

9. Huygh J, Peeters Y, Bernards J, Malbrain ML. Hemodynamic monitoring in the critically ill: an overview of current cardiac output monitoring methods. F1000Res. 2016.doi: https://doi.org/10.12688/f1000research.8991.1. eCollection 2016. Review. PubMed PMID: 8003877; PubMed Central PMCID: PMC5166586.

10. Kroll RR, McKenzie ED, Boyd JG, Sheth P, Howes D, Wood M, et al. Use of wearable devices for post-discharge monitoring of ICU patients: a feasibility study. J Intensive Care. 2017 Nov 21:5:64.

11. Stark AP, Maciel RC, Sheppard W, Sacks G, Hines OJ. An early warning score predicts risk of death after in-hospital cardiopulmonary arrest in surgical patients. Am Surg. 2015 Oct;81(10):916-21.

12. Wacker J, Staender S. The role of the anesthesiologist in perioperative patient safety. Curr Opin Anaesthesiol. 2014 Dec;27(6):649-56.

13. Wright SM. Patient safety in anesthesia: learning from the culture of highreliability organizations. Crit Care Nurs Clin North Am. 2015 Mar;27(1):1-16.

14. Krombach JW, Marks JD, Dubowitz G, Radke OC. Development and implementation of checklists for routine anesthesia care: a proposal for improving patient safety. Anesth Analg. 2015 Oct;121(4):1097-103.

15. Apfelbaum JL, Hagberg CA, Caplan RA, Blitt CD, Connis RT, Nickinovich DG, et al. Practice guidelines for management of the difficult airway: an updated report by the American Society of Anesthesiologists Task Force on Management of the Difficult Airway. Anesthesiology. 2013 Feb;118(2): 251-70.

16. Merchant R, Chartrand D, Dain S, Dobson G, Kurrek MM, Lagacé A, et al. Guidelines to the practice of anesthesia - revised edition 2016. Can J Anaesth. 2016 Jan;63(1):86-112.

17. Hamzaoui O, Monnet X, Teboul JL. Evolving concepts of hemodynamic monitoring for critically ill patients. Indian J Crit Care Med. 2015 Apr;19(4): 220-6.

18. Arriaga AF, Bader AM, Wong JM, Lipsitz SR, Berry WR, Ziewacz JE, et al. Simulation-based trial of surgical-crisis checklists. N Engl J Med. 2013 Jan 17; 368(3):246-53.

19. Low D, Walker I, Heitmiller ES, Kurth D. Implementing checklists in the operating room. Paediatr Anaesth. 2012 Oct;22(10):1025-31.

20. Rosenfield LK, Chang DS. The error of omission: a simple checklist approach for improving operating room safety. Plast Reconstr Surg. 2009 Jan;123(1): 399-402.

21. Boat AC, Spaeth JP. Handoff checklists improve the reliability of patient handoffs in the operating room and postanesthesia care unit. Paediatr Anaesth. 2013 Jul:23(7):647-54

22. Erestam S, Haglind E, Bock D, Andersson AE, Angenete E. Changes in safety climate and teamwork in the operating room after implementation of a revised WHO checklist: a prospective interventional study. Patient Saf Surg. 2017 Jan 31;11:4.

23. Haynes AB, Weiser TG, Berry WR, Lipsitz SR, Breizat AH, Dellinger EP, et al. A surgical safety checklist to reduce morbidity and mortality in a global population. N Engl J Med. 2009 Jan 29;360(5):491-9.

24. McGill J. Airway management in trauma: an update. Emerg Med Clin North Am. 2007;25(3):603-22. vii

25. Frova G, Sorbello M. Algorithms for difficult airway management: a review. Minerva Anestesiol. 2009 Apr;75(4):201-9.

26. Berkow LC, Morey TE, Urdaneta F. The technology of video laryngoscopy. Anesth Analg. 2017. doi: https://doi.org/10.1213/ANE.0000000000002490. [Epub ahead of print] PubMed PMID: 28961559.

27. Bjoernsen LP, Lindsay B. Video laryngoscopy in the prehospital setting. Prehosp Disaster Med. 2009 Jun;24(3):265-70. 
28. Aziz MF, Kim D, Mako J, Hand K, Brambrink AM. A retrospective study of the performance of video laryngoscopy in an obstetric unit. Anesth Analg. 2012 Oct;115(4):904-6.

29. Cheyne DR, Doyle P. Advances in laryngoscopy: rigid indirect laryngoscopy. F1000 Med Rep. 2010 Aug 19;2:61.

30. Russo SG, Weiss M, Eich C. Video laryngoscopy olé! Time to say good bye to direct and flexible intubation? Anaesthesist. 2012 Dec;61(12):1017-26.

31. Xue FS, Liu GP, Sun C, Li RP. Comparing emergency intubation with direct and video laryngoscopy. Acad Emerg Med. 2016 May 14;23(6):747-8.

32. Mort TC. Continuous airway access for the difficult extubation: the efficacy of the airway exchange catheter. Anesth Analg. 2007;105(5):1357-62. table of contents

33. Cooper RM. The use of an endotracheal ventilation catheter in the management of difficult extubations. Can J Anaesth. 1996 Jan;43(1):90-3.

34. Moyers G, McDougle L. Use of the cook airway exchange catheter in "bridging" the potentially difficult extubation: a case report. AANA J. 2002 Aug;70(4):275-8.

35. Gordon J, Cooper RM, Parotto M. Supraglottic airway devices: indications, contraindications and management. Minerva Anestesiol. 2018;84(3):389-97. doi: https://doi.org/10.23736/S0375-9393.17.12112-7. Epub 2017 Oct 12. PubMed PMID: 29027772.

36. Hernandez MC, Aho JM, Zielinski MD, Zietlow SP, Kim BD, Morris DS. Definitive airway management after pre-hospital supraglottic airway insertion: outcomes and a management algorithm for trauma patients. Am J Emerg Med. 2018 Jan;36(1):114-9.

37. Huang AS, Hajduk J, Jagannathan N. Advances in supraglottic airway devices for the management of difficult airways in children. Expert Rev Med Devices. 2016 Jan 29;13(2):157-69.

38. Vithalani VD, VIk S, Davis SQ, Richmond NJ. Unrecognized failed airway management using a supraglottic airway device. Resuscitation. 2017 Oct; 119:1-4.

39. Katsnelson Y, Raman J, Katsnelson F, Mor-Avi V, Heller LB, Jayakar D, et al. Current state of intraoperative echocardiography. Echocardiography. 2003 Nov;20(8):771-80.

40. Pislaru SV, Michelena HI, Mankad SV. Interventional echocardiography. Prog Cardiovasc Dis. 2014 Aug;57(1):32-46.

41. Stock KF, Klein B, Steubl D, Lersch C, Heemann U, Wagenpfeil S, et al. Comparison of a pocket-size ultrasound device with a premium ultrasound machine: diagnostic value and time required in bedside ultrasound examination. Abdom Imaging. 2015 Oct;40(7):2861-6.

42. Kobal SL, Trento L, Baharami S, Tolstrup K, Naqvi TZ, Cercek B, et al. Comparison of effectiveness of hand-carried ultrasound to bedside cardiovascular physical examination. Am J Cardiol. 2005 Oct 1;96(7):1002-6.

43. Galusko V, Khanji MY, Bodger O, Weston C, Chambers J, Ionescu A. Handheld ultrasound scanners in medical education: a systematic review. J Cardiovasc Ultrasound. 2017 Sep 29;25(3):75-83.

44. Kimura BJ, Shaw DJ, Amundson SA, Phan JN, Blanchard DG, DeMaria AN. Cardiac limited ultrasound examination techniques to augment the bedside cardiac physical examination. J Ultrasound Med. 2015 Sep;34(9):1683-90.

45. Smith ME, Chiovaro JC, O'Neil M, Kansagara D, Quiñones AR, Freeman M, et al. Early warning system scores for clinical deterioration in hospitalized patients: a systematic review. Ann Am Thorac Soc. 2014 Nov;11(9):1454-65.

46. Friedman AM. Maternal early warning systems. Obstet Gynecol Clin N Am. 2015 Jun;42(2):289-98.

47. Paliwoda M, New K, Bogossian F. Neonatal early warning tools for recognising and responding to clinical deterioration in neonates cared for in the maternity setting: a retrospective case-control study. Int J Nurs Stud. 2016 Sep;61:125-35.

48. Parfitt SE, Bogat ML, Hering SL, Ottley C, Roth C. Sepsis in obstetrics: clinical features and early warning tools. MCN Am J Matern Child Nurs. 2017;42(4):199-205.

49. Miranda JOF, Camargo CL, Nascimento CL, Portela DS, Monaghan A Accuracy of a pediatric early warning score in the recognition of clinical deterioration. Rev Lat Am Enfermagem. 2017 Jul 10;25:e2912.

50. Hasselmann K, Munk W, MacDonald G. Bispectra of ocean waves. In: Rosenblatt M, editor. Time series analysis; 1963. p. 125-39.

51. Haubrich RA. Earth noise 5 to 500 millicycles per second. J Geophys Res. 1965;70:1415-27.

52. Sigl JC, Chamoun NG. An introduction to bispectral analysis for the electroencephalogram. J Clin Monit. 1994 Nov;10(6):392-404.

53. Vuyk J, Lichtenbelt BJ, Vieveen J, Dahan A, Engbers FH, Burm AG. Low bispectral index values in awake volunteers receiving a combination of propofol and midazolam. Anesthesiology. 2004 Jan 1;100(1):179-81.
54. Edwards JJ, Soto RG, Thrush DM, Bedford RF. Bispectral index scale is higher for halothane than sevoflurane during intraoperative anesthesia. Anesthesiology. 2003 Dec;99(6):1453-5.

55. Davidson AJ, Czarnecki C. The Bispectral index in children: comparing isoflurane and halothane. $\mathrm{Br} J$ Anaesth. 2004 Jan;92(1):14-7.

56. Vereecke HE, Struys MM, Mortier EPA. Comparison of bispectral index and ARX-derived auditory evoked potential index in measuring the clinical interaction between ketamine and propofol anaesthesia. Anaesthesia. 2003 Oct:58(10):957-61.

57. Rampil IJA. Primer for EEG signal processing in anesthesia. Anesthesiology. 1998 Oct:89(4):980-1002.

58. Dahaba AA. Different conditions that could result in the bispectral index indicating an incorrect hypnotic state. Anesth Analg. 2005 Sep;101(3):765-73.

59. Fuchs-Buder T, Claudius C, Skovgaard LT, Eriksson LI, Mirakhur RK, VibyMogensen J, et al. Good clinical research practice in pharmacodynamic studies of neuromuscular blocking agents II: the Stockholm revision. Acta Anaesthesiol Scand. 2007 Aug;51(7):789-808.

60. Dahaba AA, Von Klobucar F, Rehak PH, List WF. Comparison of a new piezoelectric train-of-four neuromuscular monitor, the ParaGraph, and the Relaxometer mechanomyograph. Br J Anaesth. 1999 May;82(5):780-2.

61. Dahaba AA, von Klobucar F, Rehak PH, List WF. The neuromuscular transmission module versus the relaxometer mechanomyograph for neuromuscular block monitoring. Anesth Analg. 2002;94(3):591-6. table of contents

62. Loan PB, Paxton LD, Mirakhur RK, Connolly FM, McCoy EP. The TOF-guard neuromuscular transmission monitor. A comparison with the Myograph 2000. Anaesthesia. 1995 Aug;50(8):699-702.

63. Claudius C, Viby-Mogensen J. Acceleromyography for use in scientific and clinical practice: a systematic review of the evidence. Anesthesiology. 2008 Jun;108(6):1117-40.

64. Cosper P, Zellinger M, Enebo A, Jacques S, Razzano L, Flack MN. Improving clinical alarm management: guidance and strategies. Biomed Instrum Technol. 2017 Apr;51(2):109-15.

65. Ronquillo JG, Zuckerman DM. Software-related recalls of health information technology and other medical devices: implications for FDA regulation of digital health. Milbank Q. 2017;95(3):535-53.

66. Ergonomics DD. Patient safety, and engineering ethics: a case study and cautionary tale. J Long-Term Eff Med Implants. 2007;17(1):27-33.

67. Bennett KB. Ecological interface design and system safety: one facet of Rasmussen's legacy. Appl Ergon. 2017 Mar;59(Pt B):625-36.

68. Israelski EW, Muto WH. Human factors risk management as a way to improve medical device safety: a case study of the Therac 25 radiation therapy system. Jt Comm J Qual Saf. 2004 Dec;30(12):689-95.

69. Doyle DJ. Consumer reports for anesthesia equipment: an idea whose time has come? Anesth Analg. 2007;105(6):1866-7. author reply 1867

Ready to submit your research? Choose BMC and benefit from:

- fast, convenient online submission

- thorough peer review by experienced researchers in your field

- rapid publication on acceptance

- support for research data, including large and complex data types

- gold Open Access which fosters wider collaboration and increased citations

- maximum visibility for your research: over $100 \mathrm{M}$ website views per year

At BMC, research is always in progress.

Learn more biomedcentral.com/submissions 